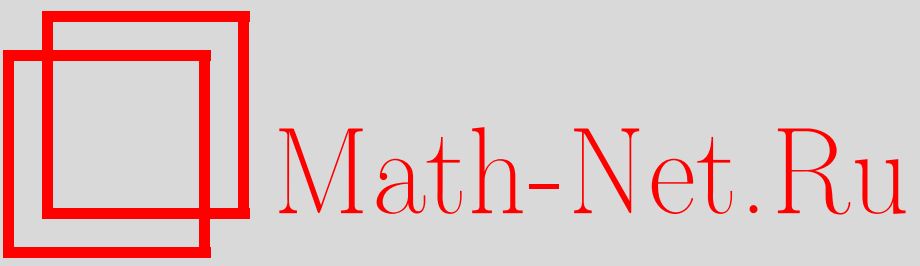

Ю. С. Вернов, М. Н. Мнацаканова, Дисперсионные соотношения для амплитуды упругого рассеяния вперед в некоммутативной квантовой теории поля, ТМФ, 2004, том 139, номер 1, 3-11

DOI: https://doi.org/10.4213/tmf49

Использование Общероссийского математического портала Math-Net.Ru подразумевает, что вы прочитали и согласны с пользовательским соглашением

http://www.mathnet.ru/rus/agreement

Параметры загрузки:

IP : 54.224 .135 .184

26 апреля 2023 г., 09:55:00 
ТЕОРЕТИЧЕСКАЯ

И МАТЕМАТИЧЕСКАЯ

ФИЗИКА

Том 139, № 1

апрель, 2004

(C) 2004 г. $\quad$ Ю.С. Вернов* ${ }^{*}$ М.Н. Мнацаканова ${ }^{\dagger}$

\section{ДИСПЕРСИОННЫЕ СООТНОШЕНИЯ ДЛЯ АМПЛИТУДЫ УПРУГОГО РАССЕЯНИЯ ВПЕРЕД В НЕКОММУТАТИВНОЙ КВАНТОВОЙ ТЕОРИИ ПОЛЯ}

Доказано, что в некоммутативной квантовой теории поля амплитуда упругого рассеяния вперед двух массивных бесспиновых частиц имеет те же аналитические свойства, что и в коммутативном случае, если условие некоммутативности задано только для пространственных переменных.

Ключевые слова: некоммутативная квантовая теория поля, локальная коммутативность, аналитичность, дисперсионные соотношения.

\section{1. ВВЕДЕНИЕ}

Доказательство аналитических свойств амплитуд рассеяния - одно из наиболее важных достижений аксиоматической квантовой теории поля. Дисперсионные соотношения (ДС), полученные в [1], впервые были строго доказаны в работе Боголюбова [2]. Другие доказательства даны в работах [3]; подробный вывод ДС для $(\pi-N)$-рассеяния приведен в книге [4].

В настоящее время широко исследуются обобщения стандартной квантовой теории поля. Некоммутативная квантовая теория поля, имеющая в своей основе некоммутативную геометрию, созданную Конном [5], рассматривается сейчас как весьма перспективный вариант такого обобщения (см. обзоры [6]).

Поэтому очень интересно исследовать проблему аналитичности в такой теории. В наиболее популярном варианте некоммутативной квантовой теории предполагаются справедливыми следующие коммутационные соотношения для координат:

$$
\left[x_{\mu}, x_{\nu}\right]=i \theta_{\mu \nu}
$$

где постоянные $\theta_{\mu \nu}$ образуют антисимметричную матрицу. Эти соотношения означают, что взаимодействие носит нелокальный характер, поскольку условие (1) эквивалентно замене в обычном пространстве произведения операторов на новое мояловское произведение (см., например, [6]), содержащее производные всех порядков.

\footnotetext{
${ }^{*}$ Институт ядерных исследований РАН, Москва, Россия. E-mail: vernov@ms2.inr.ac.ru

${ }^{\dagger}$ Научно-исследовательский институт ядерной физики Московского государственного университета, Москва, Россия. E-mail: mnatsak@theory.sinp.msu.ru
} 
Известно, что ключевую роль в доказательстве аналитических свойств амплитуд рассеяния в коммутативном случае играет условие локальной коммутативности (или эквивалентное ему условие микропричинности Боголюбова). В некоммутативной теории у нас нет оснований считать эти условия выполненными. В работах [7], [8] показано, что в общем варианте нелокальной теории, когда $\theta_{0} \neq 0$, микропричинность отсутствует. В этом случае в теории возникают также проблемы с макропричинностью [9].

Однако если некоммутативность затрагивает только пространственные переменные $\left(\theta_{0 i}=0, \quad i=1,2,3\right)$, то можно сформулировать (см. ниже формулы $(2)$ и $\left.(3)\right)$ аналог условия локальной коммутативности для некоммутативной теории [7].

Отметим, что условие (3) требует обрашения в нуль коммутатора токов лишь на подмножестве того множества точек, в которых он равен нулю в стандартной теории. Кроме того, редукционные формулы Лемана-Симанзика-Циммермана (ЛСЦ), как и аналогичные формулы в формализме Боголюбова-Медведева-Поливанова, являюшиеся базисом для вывода аналитических свойств амплитуд рассеяния, могут претерпеть существенные изменения в обшем случае, но остаются справедливыми, если некоммутативность затрагивает только пространственные переменные. Поэтому мы ограничимся вариантом некоммутативной теории с $\theta_{0 i}=0$.

Можно ожидать, что аналитичность сохранится в таком варианте некоммутативной теории, если ослабленного условия локальной коммутативности достаточно для вьвода аналитических свойств амплитуд рассеяния.

Отметим, что именно в том случае, когда $\theta_{0 i}=0$, соответствующий вариант некоммутативной теории может быть получен как низкоэнергетический предел теории струн [10], что делает этот вариант теории весьма привлекательным. В этом варианте был развит не только лагранжев подход, основанный на замене обычного произведения полей мояловским, но и более обший, в основе которого лежат функции Вайтмана [11].

Первый шаг в изучении аналитических свойств амплитуд рассеяния в рассматриваемом варианте теории был сделан в работе Льяо и Сиболда [12], где изучалось рассеяние вперед безмассовой частишы на массивной. Основной вывод этой работы о сушественном отличии аналитических свойств амплитуды рассеяния в коммутативном и некоммутативном случаях связан с конкретным способом продолжения амплитуды рассеяния в комплексную плоскость.

В настоящей работе мы рассмотрим аналитические свойства амплитуды упругого рассеяния двух массивных частиц для наиболее простого и одновременно наиболее важного случая рассеяния вперед. Отметим, что для рассеяния вперед ДС для $(\pi-N)$-рассеяния после усреднения по спинам совпадают с ДС для бесспиновых частиц с теми же массами. Поэтому мы ограничимся изучением упругого рассеяния бесспиновых частиц с массами $m$ ( " $\pi$-мезон") и $M$ ("нуклон"), считая $\pi$-мезон нейтральной частищей. Это означает, что наши результаты справедливы для $\left(\pi^{0}-N\right)$-рассеяния или для симметричной амплитуды (суммы амплитуд $\left(\pi^{+}-N\right)$ - и $\left(\pi^{-}-N\right)$-рассеяния). Из проведенного ниже анализа видно, что аналогичные выводы справедливы и для $\left(\pi^{ \pm}-N\right)$-рассеяния.

Как будет показано в работе, ослабленного условия локальной коммутативности достаточно для того, чтобы амплитуда упругого рассеяния вперед имела обычные анали- 
тические свойства по энергетической переменной.

Подчеркнем, что возможность доказательства аналитичности при более общем условии, чем локальная коммутативность, является важной и вне связи с некоммутативным вариантом квантовой теории поля. Естественно ожидать, что при сверхвысоких энергиях мы будем иметь дело с новой теорией, поэтому представляет интерес доказательство аналитических свойств амплитуды рассеяния при более общих предположениях, чем используемые в стандартном варианте теории.

\section{2. ДОКАЗАТЕЛЬСТВО ДС}

Как известно, в стандартной теории условие локальной коммутативности для токов имеет вид

$$
[j(x), j(y)]=0 \text { при }(x-y)^{2}<0 .
$$

В некоммутативной теории, когда $\theta_{0 i}=0$, можно выбрать систему координат таким образом, что только $\theta_{12}=-\theta_{21} \neq 0$ (см., например, [12]). В направлении, перпендикулярном плоскости некоммутативности, применимы те же соображения, что используются при постулировании (2). Поэтому естественно считать, что

$$
[j(x), j(y)]=0 \text { при }\left(x_{0}-y_{0}\right)^{2}-\left(x_{3}-y_{3}\right)^{2}<0 .
$$

Это условие было введено в работе [7].

Асимптотические поля в некоммутативной теории могут быть построены тем же образом, что и в коммутативной. Условие $\theta_{0 i}=0$ позволяет провести стандартные рассуждения, необходимые для вывода редукционных формул ЛСЦ (см., например, [13]). Согласно этим формулам амплитуда упругого рассеяния вперед бесспиновых частиц $c$ массами $m$ и $M$ имеет вид

$$
F(E, \vec{q})=\int d^{4} x e^{i\left(E x_{0}-\vec{q} \vec{x}\right)} \tau\left(x_{0}\right) F(x),
$$

где

$$
F(x)=\left\langle M\left|\left[j\left(\frac{x}{2}\right), j\left(-\frac{x}{2}\right)\right]\right| M\right\rangle, \quad j(x) \equiv\left(\square+m^{2}\right) \varphi(x),
$$

$\tau\left(x_{0}\right)$ - известная ступенчатая функция: $\tau\left(x_{0}\right)=1, x_{0} \geqslant 0, \tau\left(x_{0}\right)=0, x_{0}<0$.

Соотношение (4) записано в системе покоя нуклона, $E$ и $\vec{q}-$ энергия и импульс мезона. Интеграл по некоммутируюшим переменным сушествует в принятом в некоммутативной геометрии смысле.

ЗАМЕЧАНИЕ 1 . В действительности, как и в коммутативном случае, $F(x)$ содержит дополнительный член

$$
\delta\left(x_{0}\right)\left\langle M\left|\left[j\left(\frac{x}{2}\right), \frac{\partial}{\partial x_{0}} \varphi\left(-\frac{x}{2}\right)\right]\right| M\right\rangle,
$$

который, однако, не изменяет аналитических свойств $F(E, \vec{q})$, поскольку на основании тех же аргументов, что и в стандартном случае, является полиномом по $E$ (см. [13], гл. 12). 
Подчеркнем, что амплитуда рассеяния вперед является функцией только энергии. Однако мы не опускаем ее формальную зависимость от импульса, поскольку для распространения этой амплитуды на комплексные значения $E(\operatorname{Im} E>0)$ используется специальное представление, в которое компоненты импульса входят несимметричным образом. А именно,

$$
\begin{aligned}
F(E, \vec{q})=F\left(E, \pm\left|q_{3}\right|\right) & =\int_{0}^{\infty} e^{i E x_{0}} d x_{0} \int_{-x_{0}}^{x_{0}} e^{\mp i x_{3} \sqrt{E^{2}-E_{0}^{2}}} \Phi\left(x_{0}, x_{3}\right) d x_{3} \\
\Phi\left(x_{0}, x_{3}\right) & =\int F(x) e^{-i\left(q_{1} x_{1}+q_{2} x_{2}\right)} d x_{1} d x_{2} \\
E_{0}^{2} & =m^{2}+q_{1}^{2}+q_{2}^{2}, \quad q_{3}= \pm \sqrt{E^{2}-E_{0}^{2}}
\end{aligned}
$$

(аналогичная процедура была проведена в работе [12]). При написании равенства (5) мы учли условие (3). Для простоты зависимость функции $\Phi\left(x_{0}, x_{3}\right)$ от $q_{1}$ и $q_{2}$ опущена. Мы докажем, что при любых фиксированных $q_{1}$ и $q_{2}$ амплитуда рассеяния является одной и той же аналитической функцией, определяемой соотношением (21) (см. ниже).

Мы можем исключить сингулярность $\sqrt{E^{2}-E_{0}^{2}}$, перейдя к $F(E)=\left(F\left(E,+\left|q_{3}\right|\right)+\right.$ $\left.F\left(E,-\left|q_{3}\right|\right)\right) / 2$. В соответствии с равенством (5)

$$
F(E)=\int_{0}^{\infty} e^{i E x_{0}} d x_{0} \int_{-x_{0}}^{x_{0}} \cos \left(x_{3} \sqrt{E^{2}-E_{0}^{2}}\right) \Phi\left(x_{0}, x_{3}\right) d x_{3}
$$

Непосредственное распространение $F(E)$ на комплексные $E$ невозможно, поскольку

$$
\operatorname{Im} \sqrt{E^{2}-E_{0}^{2}}>\operatorname{Im} E
$$

(см. [14], гл. 10).

Чтобы преодолеть эту трудность, заменим, следуя Боголюбову и Ширкову $[14], F(E)$ на регуляризованную амплитуду $F_{\varepsilon}(E)$ :

$$
F_{\varepsilon}(E)=\int_{0}^{\infty} e^{i E x_{0}} d x_{0} \int_{-x_{0}}^{x_{0}} \cos \left(x_{3} \sqrt{E^{2}-E_{0}^{2}}\right) e^{-\varepsilon\left(x_{0}^{2}+x_{3}^{2}\right)} \Phi\left(x_{0}, x_{3}\right) d x_{3} .
$$

Вследствие сходимости интеграла в $(5 б) F_{\varepsilon}(E)$ - аналитическая функция $E$ в верхней полуплоскости.

Для доказательства аналитичности $F(E)$ мы докажем, что существует $\lim _{\varepsilon \rightarrow 0} F_{\varepsilon}(E)$, которьй является аналитической функцией. С этой целью представим $F_{\varepsilon}(E)$ в виде интеграла по вешественной оси. Однако поскольку $F_{\varepsilon}(E) \not \rightarrow 0$ при $E \rightarrow \infty$, сначала надо 
построить вспомогательную функцию $\psi_{\varepsilon}(E)$ такую, что $\psi_{\varepsilon}(E) \rightarrow 0, E \rightarrow \infty$. Это можно сделать, если сушествует $n$ такое, что при $E \rightarrow \infty$

$$
\frac{F(E)}{E^{n}} \rightarrow 0, \quad \frac{F_{\varepsilon}(E)}{E^{n}} \rightarrow 0 .
$$

В коммутативном случае $n=2$ вследствие ограничения Фруассара-Мартена $|F(E)|<$ $C E \ln ^{2} E$. Поскольку это ограничение не доказано в некоммутативном случае, мы исходим из более общего условия.

Заметим, что вследствие эрмитовости $j(x)$ ( $\varphi(x)$ - действительное поле) $F(E)$, а следовательно, и $F_{\varepsilon}(E)$, удовлетворяют условию кроссинг-симметрии

$$
F_{\varepsilon}(-E+i 0)=F_{\varepsilon}^{*}(E+i 0) .
$$

Согласно условиям (6) и (7)

$$
\psi_{\varepsilon}(E) \rightarrow 0, \quad E \rightarrow \pm \infty
$$

если

$$
\psi_{\varepsilon}(E)=\frac{F_{\varepsilon}(E)}{\prod_{1}^{n}\left(E-E_{i}\right)}, \quad E_{i}>E_{0} .
$$

Воспользуемся формулой Коши

$$
\psi_{\varepsilon}(E)=\frac{1}{2 \pi i} \int_{C} \frac{\psi_{\varepsilon}\left(E^{\prime}\right) d E^{\prime}}{E^{\prime}-E}, \quad \operatorname{Im} E>0 .
$$

Контур $C$ состоит из отрезка $[-R, R]$, в котором отрезки $\left[E_{i}-\varepsilon, E_{i}+\varepsilon\right]$ заменены соответствующими полуокружностями, и полуокружности радиуса $R$ в верхней полуплоскости.

Покажем, что вследствие условий (3) и (8)

$$
\psi_{\varepsilon}\left(R e^{i \varphi}\right) \rightarrow 0
$$

при $R \rightarrow \infty, 0<\varphi<\pi$.

Действительно, если $|E| \rightarrow \infty$, то $\operatorname{Im} \sqrt{E^{2}-E_{0}^{2}} \cong \operatorname{Im} E-\operatorname{Im} E_{0}^{2} /(2 E)$ и, следовательно,

$$
\left|e^{i E x_{0}} \cos \left(x_{3} \sqrt{E^{2}-E_{0}^{2}}\right)\right| \leqslant e^{-\operatorname{Im} E\left(x_{0}-x_{3}\right)} e^{E_{0}^{2} x_{3}(\varepsilon) \sin \varphi / R} .
$$

Благодаря множителю $e^{-\varepsilon x_{3}^{2}}$ интеграл по $x_{3}$ сходится, когда $x_{0} \rightarrow \infty$, и реально область интегрирования сводится к $\left(-x_{3}(\varepsilon), x_{3}(\varepsilon)\right)$. Согласно неравенству (11) при любом фиксированном $\varepsilon$ и $R \rightarrow \infty$ экспоненшиально растуший фактор в (5б) исчезает, и поэтому из условия (8) следует условие (10). 
ЗАмечаниЕ 2. Согласно теореме Фрагмена-Линделефа (см., например, [15]) на самом деле условие (10) - следствие условия (8) для всех функций, аналитических в верхней полуплоскости и возрастаюших при $R \rightarrow \infty$ слабее любой экспоненты.

Согласно условию (10), положив в (9) $R=\infty$, приходим к равенству, справедливому при любом фиксированном $\varepsilon$ :

$$
\psi_{\varepsilon}(E)=\frac{1}{2 \pi i} \int_{-\infty}^{\infty} \frac{\psi_{\varepsilon}\left(E^{\prime}\right) d E^{\prime}}{E^{\prime}-E}-\frac{1}{2} \sum_{i=1}^{n} \frac{F_{\varepsilon}\left(E_{i}\right)}{\left(E_{i}-E\right) \prod_{i \neq j}\left(E_{i}-E_{j}\right)}, \quad \operatorname{Im} E>0 .
$$

Перейдем теперь к пределу $\varepsilon=0$. Для любого члена суммы в правой части (12) этот переход очевиден. Для доказательства возможности осушествления его в интеграле рассмотрим отдельно интервалы $(-\infty,-m),(-m, m),(m, \infty)$. Сначала рассмотрим интервал $(m, \infty)$. Если $E^{\prime}>E_{0}$, мы можем перейти к пределу, воспользовавшись формулой (5б). Если $m<E^{\prime}<E_{0}$, использовать эту формулу нельзя. Однако мы можем воспользоваться тем фактом, что при физических энергиях амплитуда рассеяния вперед является функцией только энергии. Предположим, что это справедливо и для регуляризованной амплитуды. Отметим, что, хотя мы использовали конкретную регуляризацию, на самом деле вследствие условия (3) любая регуляризация, обеспечивающая сходимость интеграла в (5б) при комплексных $E$, приводит к представлению (12) для $\psi_{\varepsilon}(E)$.

Рассмотрим теперь две функции $F_{\varepsilon}^{(1)}(E)$ и $F_{\varepsilon}^{(2)}(E)$, определенные формулой $(5 б)$, но соответствующие разным значениям $q_{1}^{2}+q_{2}^{2}$ и, следовательно, разным значениям $E_{0}$ : $E_{0}^{(1)}$ и $E_{0}^{(2)}$. Пусть, например, $E_{0}^{(1)}<E_{0}^{(2)}$. Очевидно, что если $E>E_{0}^{(2)}$, то $F_{\varepsilon}^{(1)}(E)=$ $F_{\varepsilon}^{(2)}(E)$, и для каждой из них в формуле (5б) может быть осушествлен предельный переход $\lim _{\varepsilon \rightarrow 0} F_{\varepsilon}^{(1)}(E)=\lim _{\varepsilon \rightarrow 0} F_{\varepsilon}^{(2)}(E)=F(E)$. Отметим, что при этих энергиях формула (5б) совпадает с формулой (4) для регуляризованной амплитуды. При любом фиксированном $\varepsilon$ функции $F_{\varepsilon}^{(1)}(E)$ и $F_{\varepsilon}^{(2)}(E)$ аналитичны в верхней полуплоскости. Совпадая на части границы, они совпадают всюду, в том числе и в интервале $\left(E_{0}^{(1)}, E_{0}^{(2)}\right)$. Однако в этом интервале энергий для функции $F_{\varepsilon}^{(1)}(E)$ уже сушествует в формуле (5б) предельный переход к $\varepsilon=0$, так как этот интервал является физическим для функции $F_{\varepsilon}^{(1)}(E)$. Поскольку $F_{\varepsilon}^{(1)}(E)=F_{\varepsilon}^{(2)}(E)$, то соответствуюший предел сушествует и у $F_{\varepsilon}^{(2)}(E)$. Продолжая этот процесс, придем к $E_{0}=m$, т.е. осушествим предельный переход к $\varepsilon=0$ на всем интервале $(m, \infty)$.

Подчеркнем, что коммутативный и некоммутативный случаи различаются именно наличием этого интервала, в котором невозможно непосредственное использование исходной формулы (5б).

Вследствие условия ( 7$)$ в интервале $(-\infty,-m)$ можно перейти к пределу аналогично тому, как это сделано в интервале $(m, \infty)$.

Покажем, что оставшийся интервал $(-m, m)$ может быть рассмотрен так же, как и в коммутативном случае [14]. Введем функцию

$$
\widetilde{F}(E, \vec{q})=\int d^{4} x e^{i\left(E x_{0}-\vec{q} \vec{x}\right)} \tau\left(-x_{0}\right) F(-x) .
$$


Соответствуюшая ей регуляризованная функция имеет вид

$$
\widetilde{F}_{\varepsilon}(E)=\int d^{4} x \cos (\vec{q} \vec{x}) \tau\left(-x_{0}\right) e^{-\varepsilon\left(x_{0}^{2}+x_{3}^{2}\right)} F(-x) .
$$

Функцию $\widetilde{F}_{\varepsilon}(E)$ можно записать в виде, аналогичном формуле (5б):

$$
\widetilde{F}_{\varepsilon}(E)=\int_{0}^{\infty} e^{i E x_{0}} d x_{0} \int_{-x_{0}}^{x_{0}} \cos \left(x_{3} \sqrt{E^{2}-E_{0}^{2}}\right) e^{-\varepsilon\left(x_{0}^{2}+x_{3}^{2}\right)} \widetilde{\Phi}\left(x_{0}, x_{3}\right) d x_{3},
$$

где

$$
\widetilde{\Phi}\left(x_{0}, x_{3}\right)=\int F(-x) e^{-i\left(q_{1} x_{1}+q_{2} x_{2}\right)} d x_{1} d x_{2} .
$$

На основе приведенных ранее аргументов $\widetilde{F}_{\varepsilon}(E)$ аналитична в нижней полуплоскости. В соответствии с равенством (7)

$$
\widetilde{F}_{\varepsilon}(E-i 0)=F_{\varepsilon}(-E+i 0)=F_{\varepsilon}^{*}(E+i 0) .
$$

Следовательно, функция

$$
\widetilde{\psi}_{\varepsilon}(E)=\frac{\widetilde{F}_{\varepsilon}(E)}{\prod_{1}^{n}\left(E-E_{i}\right)}, \quad E_{i}>E_{0}
$$

аналитична в нижней полуплоскости, и $\widetilde{\psi}_{\varepsilon}(E) \rightarrow 0, E \rightarrow \pm \infty$. Воспользуемся равенством

$$
\frac{1}{2 \pi i} \int_{\widetilde{C}} \frac{\widetilde{\psi}_{\varepsilon}\left(E^{\prime}\right) d E^{\prime}}{E^{\prime}-E}=0, \quad \operatorname{Im} E>0 .
$$

Контур $\widetilde{C}$ в нижней полуплоскости аналогичен контуру $C$ из формулы (9).

Складывая равенства (9) и (17), получим

$$
\begin{aligned}
\psi_{\varepsilon}(E)= & \frac{1}{\pi} \int_{m}^{\infty} \frac{\operatorname{Im} \psi_{\varepsilon}\left(E^{\prime}\right) d E^{\prime}}{E^{\prime}-E}+\frac{1}{\pi} \int_{-\infty}^{-m} \frac{\operatorname{Im} \psi_{\varepsilon}\left(E^{\prime}\right) d E^{\prime}}{E^{\prime}-E}- \\
& -\sum_{i=1}^{n} \frac{\operatorname{Re} F_{\varepsilon}\left(E_{i}\right)}{\left(E_{i}-E\right) \prod_{i \neq j}\left(E_{i}-E_{j}\right)}+ \\
& +\frac{1}{2 \pi i} \int_{-m}^{m} \frac{\left(\psi_{\varepsilon}\left(E^{\prime}\right)-\widetilde{\psi}_{\varepsilon}\left(E^{\prime}\right)\right) d E^{\prime}}{E^{\prime}-E}, \quad \operatorname{Im} E>0 .
\end{aligned}
$$

В трех первых членах формулы (18) мы можем перейти к пределу $\varepsilon=0$. В оставшемся интеграле это возможно, поскольку в некоммутативной теории сохраняется условие трансляционной инвариантности, что позволяет получить в физической области выражение для $F(E, \vec{q})-\widetilde{F}(E, \vec{q})$, пригодное для распространения на нефизические $E$ $(-m<E<m)$. Непосредственно из определений (4) и (13) следует, что

$$
F(E, \vec{q})-\widetilde{F}(E, \vec{q})=F_{+}(E, \vec{q})-F_{-}(E, \vec{q}),
$$


где

$$
\begin{gathered}
F_{ \pm}(E, \vec{q})=\int d^{4} x e^{i\left(E x_{0}-\vec{q} \vec{x}\right)} F_{ \pm}(x) \\
F_{+}(x)=\left\langle M\left|j\left(\frac{x}{2},\right) j\left(-\frac{x}{2}\right)\right| M\right\rangle, \quad F_{-}(x)=F_{+}(-x) .
\end{gathered}
$$

Предположим, что, как и в коммутативном случае, векторы состояний $|p, n\rangle$, где $p$ - импульс, а $n$ обозначает остальные характеристики состояния, образуют полную систему базисных векторов. Воспользовавшись трансляционной инвариантностью и полнотой системы векторов $|p, n\rangle$, получим

$$
F_{ \pm}(E, \vec{q})=\sum_{n} \sum_{p_{n}^{0}}|\langle M|j(0)| p, n\rangle|^{2} \delta\left(p_{n}^{0}-M \mp E\right)
$$

где $p_{n}^{0}=\sqrt{M_{n}^{2}+\vec{q}^{2}}$. Выражение $(20)$ допускает продолжение в область $-m<E<m$. Отметим, что $M_{n} \geqslant M+m$ или $M_{n}=M$, поскольку у $(\pi-N)$-системы нет связанных состояний. Легко видеть, что в рассматриваемом интервале энергий лишш однонуклонное состояние дает вклад в $F_{ \pm}(E, \vec{q})$, причем $F_{ \pm}(E, \vec{q}) \neq 0$ только при $E=\mp m^{2} /(2 M)$.

Следовательно, в рассматриваемом интервале

$$
\lim _{\varepsilon=0}\left(\psi_{\varepsilon}(E)-\widetilde{\psi}_{\varepsilon}(E)\right)=0
$$

везде за исключением двух точек $\pm m^{2} /(2 M)$. Интеграл по этому интервалу обратится в нуль, если предыдушее рассмотрение применить к функциям

$$
\begin{aligned}
\Phi_{\varepsilon}(E)\left(\widetilde{\Phi}_{\varepsilon}(E)\right) & =\frac{E^{2}-m^{4} /\left(4 M^{2}\right)}{\left(E-E_{n+1}\right)\left(E-E_{n+2}\right)} \psi_{\varepsilon}(E)\left(\widetilde{\psi}_{\varepsilon}(E)\right), \\
E_{n+1}>E_{0}, & E_{n+2}>E_{0}
\end{aligned}
$$

Очевидно, что, согласно полученному представлению (см. (18)), функция $\Phi(E) \equiv$ $\lim _{\varepsilon \rightarrow 0} \Phi_{\varepsilon}(E)$ аналитична во всей плоскости с разрезами $(-\infty,-m),(m, \infty)$. Соответственно для $F(E)$ имеем стандартное представление ( $\operatorname{Im} E \neq 0, n$ - четное число)

$$
F(E)=\frac{2 E^{n}}{\pi} \int_{m}^{\infty} \frac{\operatorname{Im} F\left(E^{\prime}\right) d E^{\prime}}{\left(E^{\prime}\right)^{n-1}\left(E^{\prime 2}-E^{2}\right)}+\sum_{\substack{k=0 \\ k / 2 \in \mathbb{N}}}^{n-2} C_{k} E^{k}+\text { полюсные члены, }
$$

которое в пределе $\operatorname{Im} E=0$ представляет собой ДС с $n$ вычитаниями.

Таким образом, в любой теории, в которой справедливы редукционные формулы ЛСЦ, а условие локальной коммутативности (2) заменено условием (3), амплитуда упругого рассеяния вперед имеет те же аналитические свойства, что и в обычном случае. 


\section{Список литературы}

[1] M. Gell-Mann, M.L. Goldberger, W.E. Thirring. Phys. Rev. 1954. V. 95. P. 1612; M. L. Goldberger. Phys. Rev. 1955. V. 99. P. 979; H. Miyazawa, R. Oehme. Phys. Rev. 1955. V. 99. P. 986; R. Oehme. Phys. Rev. 1955. V. 100. P. 1503; 1956. V. 102. P. 1174; M. L. Goldberger, Y. Nambu, R. Oehme. Ann. Phys. 1956. V. 2. P. 226.

[2] H. Н. Боголюбов. Доклад на международном конгрессе по теоретической физике (Сиэттл, 1956) (неопубликовано)

[3] R. Oehme. Nuovo Cimento. 1958. V. 10. P. 1316; K. Symanzik. Phys. Rev. 1957. V. 105. P. 743; H.J. Bremermann, R. Oehme, J. G. Taylor. Phys. Rev. 1958. V. 109. P. 2178; H. Lehmann. Nuovo Cimento. 1958. V. 10. P. 579.

[4] Н. Н. Боголюбов, Б. В. Медведев, М.К. Поливанов. Вопросы теории дисперсионных соотношений. М.: Физматгиз, 1958.

[5] A. Connes. Noncommutative Geometry. New York: Academic Press, 1994.

[6] M.R. Douglas, N. A. Nekrasov. Rev. Mod. Phys. 2001. V. 73. P. 977; hep-th/0106048; R. J. Szabo. Phys. Rep. 2003. V. 378. P. 207; hep-th/0109162.

[7] L. Álvarez-Gaumé, J. L. F. Barbon, R. Zwicky. JHEP. 2001. V. 0105. P. 057; hep-th/0103069.

[8] M. Chaichian, K. Nishijima, A. Tureanu. Phys. Lett. B. 2003. V. 568. P. 146; hep-th/0209008.

[9] N. Seiberg, L. Susskind, N. Toumbas. JHEP. 2000. V. 0006. P. 044; hep-th/0005015.

[10] J. Gomis, T. Mehen. Nucl. Phys. B. 2000. V. 591. P. 265; hep-th/0005129.

[11] L. Álvarez-Gaumé, M. A. Vázquez-Mozo. Nucl. Phys. B. 2003. V. 688. P. 293; hep-th/0305093.

[12] Yi Liao, K. Sibold. Phys. Lett. B. 2002. V. 549. P. 352; hep-th/0209221.

[13] Дж. Д. Бъёркен, С. Д. Дрелл. Релятивистская квантовая теория. Т. 2. М.: Наука, 1978.

[14] Н. Н. Боголюбов, Д. В. Ширков. Введение в теорию квантованных полей. М.: Наука, 1984.

[15] Н. Н. Мейман. ЖЭТФ. 1962. Т. 43. С. 2247. 